\title{
Study on Influence of Thickness and Electromagnetic Parameter of Per- fectly Matched Layer (PML)
}

\author{
Jinzu $\mathrm{Ji}^{*}$ and Feiliang Liu
}

School of Aeronautic Science and Engineering, Beihang Universty, Beijing, China 100191

\begin{abstract}
Perfectly matched layer (PML) absorbing boundary condition (ABC) is an important technique in finitedifference time-domain (FDTD) when simulating infinite area's electromagnetic behaviour in finite area. A big enough computational area was designed in which there is no influence of reflective wave. The radiation of sinusoidal line source in this area was the benchmark for assessment of reflectivity. The total wave with boundary condition subtracted by benchmark was reflective wave. Influences of the PML absorbing layer's thickness, electromagnetic parameter distribution and loss tangent were studied via this method. The results show that 15-mesh-layer may elaborate the PML's ability and the reflectivity may be only 0.003 . Better absorbing effect can be achieved when loss tangent is distributed in second power and the corresponding loss tangent in cut off boundary is 2.5 .
\end{abstract}

Keywords: Absorbing boundary condition $(\mathrm{ABC})$, computational electromagnetics, finite-difference time-domain (FDTD), perfectly matched layer (PML).

\section{INTRODUCTION}

Finite-difference time-domain (FDTD) is a very important algorithm in computational electromagnetics and has wide application in simulating electromagnetic behaviors [1, 2]. FDTD's difference equations form is induced directly from Maxwell's equations and is very simple in mathematics for simulation of complex dielectric media [3-5]. Simulation of FDTD is very smart and can be used in simulating electromagnetic wave's scattering, radiation, absorbing behavior in antenna's radiation, radar cross section's calculation, transmission line's simulation, ionosphere's simulation and so on [6-8], and even has application in acoustics [9].

Absorbing boundary condition (ABC) is absorbing layer in the cut off boundary of calculation area when FDTD is used for simulating infinitely large area. When the electromagnetic wave is incident on the absorbing layers, there is seldom reflection. That is very like the absorbing wall of anechoic chamber. The difference is that the absorbing was fulfilled via numerical method and physical material in FDTD and anechoic chamber respectively.

Perfectly matched layer (PML) is a very important absorbing boundary condition which has good absorbing effect and simple format and the absorbing performance does not vary in different incident angle [10-12]. PML method splits the electric and magnetic field into two and each has own differential operation. When setting PML condition, the mainly consideration is about absorbing layer's thickness and media's parameter distribution. This paper studies the influence of these configurations on the absorbing effect.
Different absorbing layer's thickness and media's distribution type such as linearly, parabolic, third order and fourth order was calculated and the absorbing performance was analyzed.

Assessment method of the ABC's was designed via a benchmark in big area. Sinusoidal source radiation was simulated in huge calculation area in finite time step number and there is no reflective wave in this process. This result was used as benchmark which subtracted by radiation simulation with $\mathrm{ABC}$ and the difference was just the reflective wave.

\section{SINGLE PHASE FULL BRIDGE INVERTER}

\subsection{PML's Absorbing Boundary Condition}

Take 2, mdimensional transverse magnetic (TM) wave for study and split $E_{z}$ into $E_{z x}$ and $E_{z y}$, whose differential equation is $(1)[1,13]$.

$$
\begin{aligned}
& \varepsilon \frac{\partial E_{z x}}{\partial t}+\sigma_{x} E_{z x}=\frac{\partial H_{y}}{\partial x} \\
& \varepsilon \frac{\partial E_{z y}}{\partial t}+\sigma_{y} E_{z y}=-\frac{\partial H_{x}}{\partial y} \\
& \mu \frac{\partial H_{x}}{\partial t}+\sigma_{m y} H_{x}=-\frac{\partial\left(E_{z x}+E_{z y}\right)}{\partial y} \\
& \mu \frac{\partial H_{y}}{\partial t}+\sigma_{m x} H_{y}=\frac{\partial\left(E_{z x}+E_{z y}\right)}{\partial x}
\end{aligned}
$$

where $H_{x}, H_{y}$ is magnetic field, $\sigma_{x}, \sigma_{y}$ is electric conductance, $\sigma_{m x}, \sigma_{m y}$ is magnetic conductance. When $\sigma_{x}=\sigma_{y}$ and 


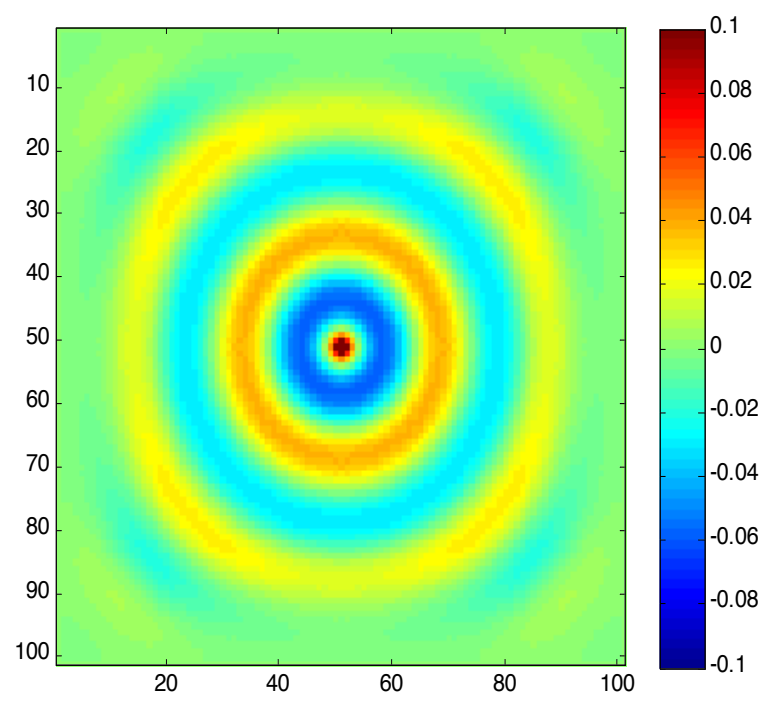

Fig. (1). Ez distribution after 200 time steps.

$\sigma_{m x}=\sigma_{m y},(1)$ is just convectional Maxwell's equation. If $\sigma_{x} \neq \sigma_{y}$ or $\sigma_{m x} \neq \sigma_{m y}$, the media is anisotropy. When specific condition is fulfilled, electromagnetic wave can transmit through the boundary of different media in any incident angle [14]. PML absorbing condition uses this characteristic and set the absorbing layer in the calculation boundary to absorbing wave. The media's parameter is often set in gradual changing arrangement style. In most outer boundary, electric and magnetic conductance is maxima and gradually descends in inner direction.

The transmitting condition in different media's boundary without reflection is $\sigma_{x} / \varepsilon=\sigma_{m x} / \mu, \sigma_{y} / \varepsilon=\sigma_{m y} / \mu[1]$.

The electromagnetic wave's angular frequency is $\omega$, electric and magnetic loss tangent is $\delta$ and $\delta_{m}$ respectively. Considering $\delta=\delta / \omega \varepsilon$ and $\delta_{m}=\delta_{m} / \omega \mu$, (2) shows that when there is no reflection, electric loss tangent and magnetic loss tangent is equal. Therefore, this paper will not discriminate these two loss tangents and take notion in $\delta$.

2-dimensional TM wave's radiation was simulated to show the PML absorbing boundary condition. The computational area's mesh number is $100 \times 100$ and the absorbing boundary layer's thickness is 20 meshes. The outer media's parameter is linearly distributed and $\delta=1$ in the most outer layer. The stimulating source is sinusoid wave whose wavelength is $1 \mathrm{~m}$. The mesh size is $1 / 20$ of wavelength. The Courant stable factor is 0.7 , that is, the time step is $\Delta t=0.7 \Delta d / c$ where $\Delta d$ is mesh size and $c$ is light speed. The source is located in the center of the computational area and the amplitude is 1 . After 200 steps' simulation, $E_{z}$ 's distribution is as Fig. (1).

In Fig. (1), the square's black outline is absorbing layer's boundary which divided the area into 2 parts. The outer is absorbing layer and the inner is free space. We can see that in absorbing layer's the electric field is attenuated along the

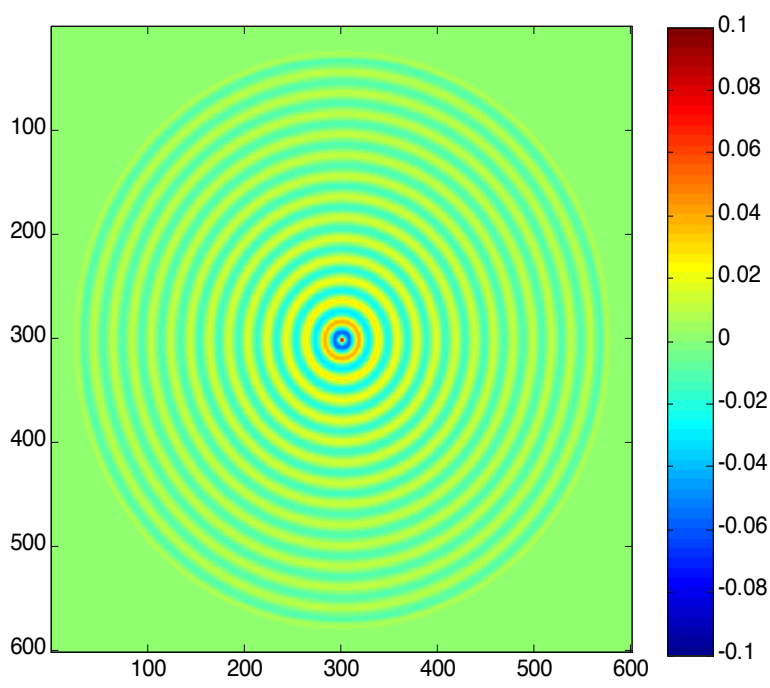

Fig. (2). Simulating result of non-reflection.

propagating direction and reduced to zeros in the cut off boundary. This absorbing mechanic is different from Mur absorbing boundary condition, which only uses one mesh thickness for absorbing wave [1].

\subsection{Assessment of Absorbing Effect}

Take fraction of reflective wave and incident wave as the relative reflectivity, short in reflectivity in this paper. The reflective wave was the subtraction of total field and radiation field without reflection which is acquired by simulation of big enough area. In a less time step number, the electromagnetic wave hasn't reach the boundary, thus there is no reflective wave in the area.

The mesh number in non-reflected area is $600 \times 600$ and the stimulating source, mesh size, time step is just the same with the former example. After simulation of 400 time steps, electromagnetic wave hasn't reach the boundary, thus there is no influence of boundary condition's reflection. The field is used as benchmark of the comparison. Take a sample point near the source 20 meshes and record the field in every time step. The sample point's location and the simulation result are in Fig. (2). The sample point's electric field versus the time step is as Fig. (3).

From Fig. (3), when the field is in steady vibrating status, the amplitude is about $0.035 \mathrm{~V} / \mathrm{m}$. In a smaller area with absorbing boundary condition, the mesh number is $100 \times 100$ and the stimulating source, mesh size, time step, simulating time step number and even the sample point's location remain the same. The subtraction of electric field in (Fig. 3) and the smaller area is the reflective field. The amplitude fraction of reflective wave and the benchmark wave 0.035 was reflectivity, denoted by $R$ in this paper.

\section{ABSORBING EFFECT OF DIFFERENT $\delta$ AND AB- SORBING LAYER THICKNESS}

The absorbing performance is better when $\delta$ is distributed in gradual format. The loss tangent is biggest in the cut 


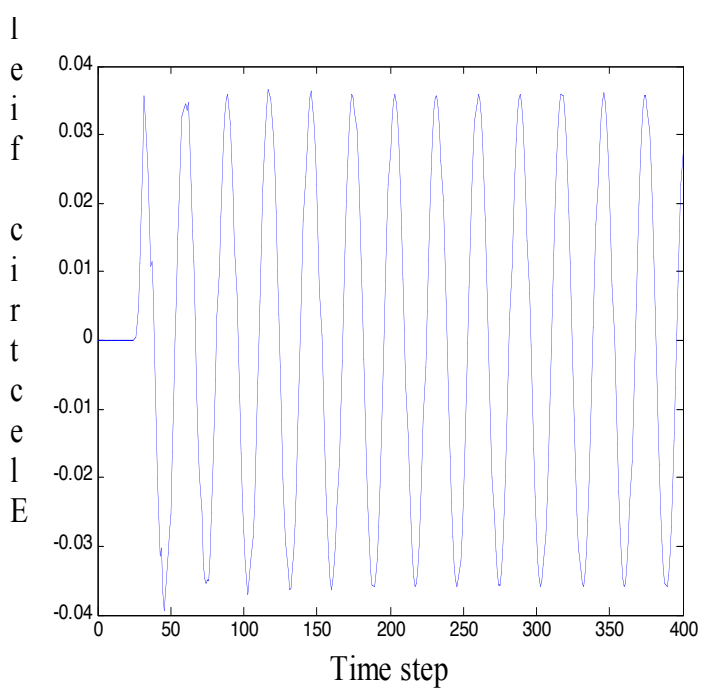

Fig. (3). Benchmark wave without reflection.

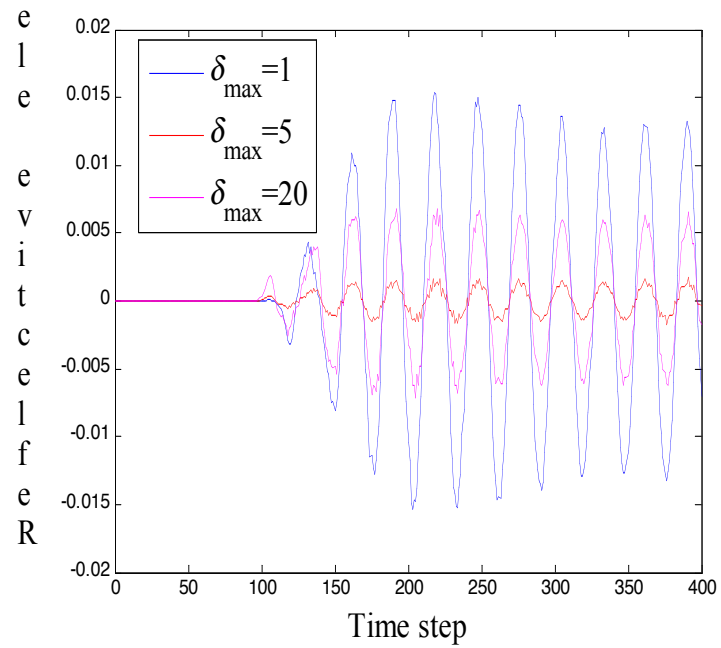

Fig. (4). Reflective wave of different $\delta$.

off boundary, denoted by $\delta_{\max }$. The inner part of the ABC gradually descends and shrinks to zero in free space. The gradual manner might be linear or second, third, fourth power. When gradual manner is defined, $\delta_{\text {max }}$ in cut off boundary defines the other electromagnetic parameter in the absorbing layer.

Assuming $\delta$ is linearly distributed and the absorbing layer's thickness is 5 meshes. Simulation was processed when the $\delta_{\max }$ is 1,5 and 20. The reflective wave was acquired via subtraction of this field with benchmark without reflection. The reflective wave's field versus time step is in Fig. (4).

From Fig. (4), different $\delta_{\max }$ has results in different absorbing effect. When $\delta_{\max }=1$, the reflective effect is the worst and the reason is that electromagnetic wave's attenuation in the absorbing layer is little, thus more electromagnetic wave transmits through the absorbing layer and imping on the cut off boundary and then reflects back. When $\delta_{\text {max }}=20$, the absorbing effect is also not good because of

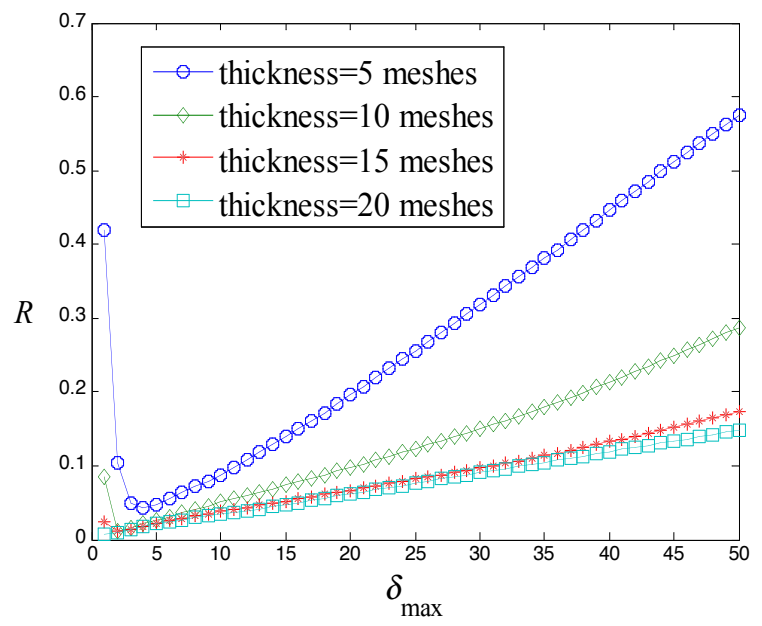

Fig. (5). $R$ versus $\delta_{\max }$.

numerical discrete influence there is reflection between absorbing layer and the inner free space. When $\delta_{\text {max }}=10$, the absorbing effect is the best of the three.

The reflectivity versus $\delta_{\text {max }}$ was in Fig. (5) of different absorbing layer's thickness. $\delta_{\max }$ is from 1 to 50 and the step is 1 .

In Fig. (5), the reflectivity $R$ versus $\delta_{\text {max }}$ first descends quickly and then ascends smoothly. For example, when the absorbing layer's thickness is 5 meshes, the absorbing effect is the best in $\delta_{\max }=4$. The absorbing effect gets worse whether $\delta_{\max }$ is bigger or smaller. The minimum reflectivity of different $\delta_{\max }$ is denoted by $R_{\min }$, then when the thickness is 5 meshes, $R_{\min }=0.05$.

In comparing absorbing layer of different thickness, the thicker of the absorbing layer, the better absorbing effect. For every thickness, there is a $\delta_{\max }$ when $R$ gets the minima value $R_{\min }$. We define this $\delta_{\text {max }}$ as best loss tangent, denoted by $\delta_{\text {cr }}$. From Figure 5 , when $\delta_{\text {max }}<\delta_{\text {cr }}$, reflectivity get smaller quickly. When $\delta_{\max }>\delta_{\text {cr }}$, the reflectivity get bigger smoothly. Thus, for a given absorbing layer's thickness, $\delta_{\text {max }}$ cannot be smaller than $\delta_{\text {cr }}$, but can a little bigger than $\delta_{\text {cr }}$.

From Fig. (5), we also see that different thickness has different $\delta_{\text {cr }}$. The thicker of absorbing layer, the smaller of $\delta_{\text {cr }}$.

Fig. (6) shows $R_{\min }$ versus $\delta_{\text {max }}$ where $\delta_{\text {max }}$ is from 0.1 to 5 and the step is 0.1. In Fig. (6) we can see more clear of the $\delta_{\text {max }}$ 's influence.

From Fig. (6), in the interval of $\delta_{\max }$ in interval of 0.1 to 5 , the thicker of the absorbing layer, the smaller of the reflectivity, that is, the absorbing effect is better. Fig. (6) shows that for every given absorbing layer's thickness there is different $\delta_{\text {cr }}$ that makes the absorbing effect gets best. $R_{\min }$ and the corresponding $\delta_{\text {cr }}$ of each thickness are shown Table 1. 


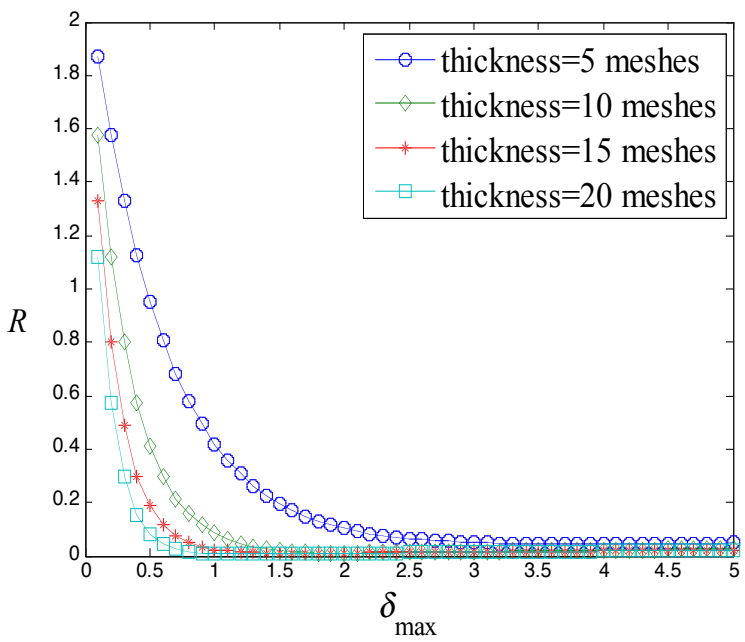

Fig. (6). $R$ versus $\delta_{\text {max }}$.

Table 1. $\boldsymbol{R}_{\min }$ and corresponding $\delta_{c r}$ of different absorbing layer's thickness.

\begin{tabular}{|c|c|c|c|c|}
\hline Absorbing Layer's Thickness & $\mathbf{5}$ & $\mathbf{1 0}$ & $\mathbf{1 5}$ & $\mathbf{2 0}$ \\
\hline \hline$R_{\min }$ & 0.0438 & 0.0103 & 0.0111 & 0.0063 \\
\hline$\delta_{\mathrm{cr}}$ & 3.8 & 1.9 & 1.7 & 1.1 \\
\hline
\end{tabular}

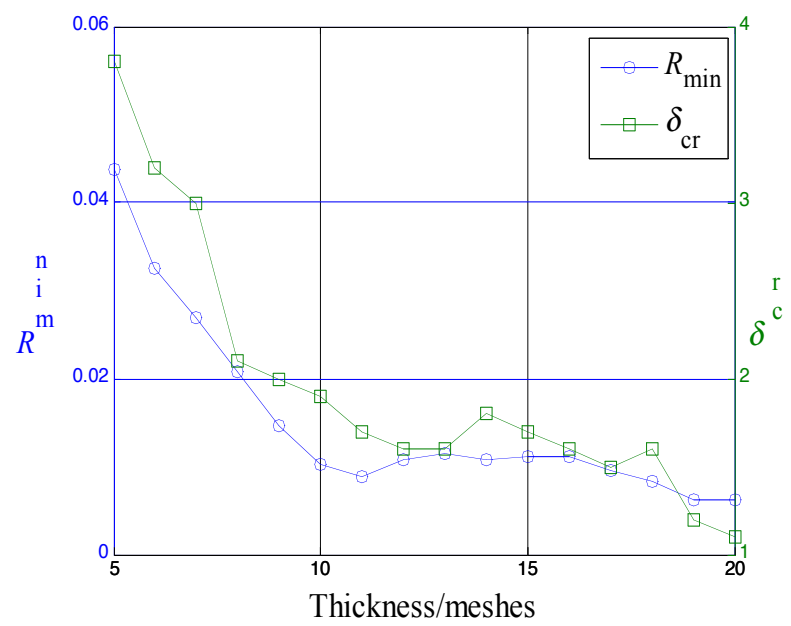

Fig. (7). $R_{\min }$ and $\delta_{\mathrm{cr}}$ versus absorbing layer's thickness.

From Table 1, the thicker of the absorbing layer, the lower of the minimum reflectivity $R_{\min }$ and the smaller of $\delta_{\text {cr }}$. Thus, raise absorbing layer's thickness can increase the absorbing effect and the corresponding $\delta_{\text {cr }}$ should be chosen.

For a study on the absorbing effect about the absorbing layer's thickness, the $R_{\text {min }}$ of the absorbing layer's thickness between 5 20 meshes and the corresponding $\delta_{\text {cr }}$ was acquired in simulation. The media's parameter distribution is still linearly distributed. The computational results are in Fig. (7).

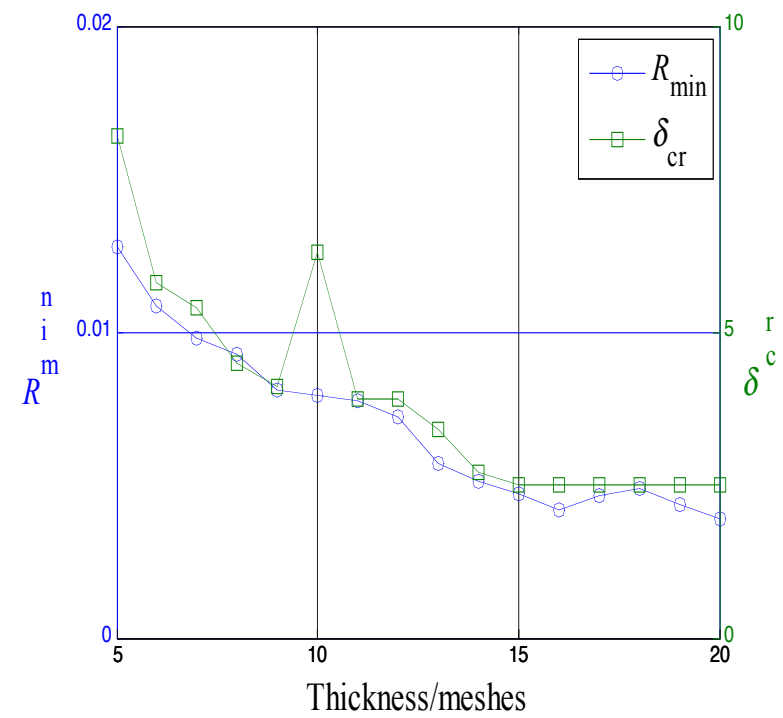

Fig. (8). $R_{\min }$ and $\delta_{\text {cr }}$ when $\delta$ is second power distributed.

From Fig. (7), as the absorbing layer's thickness increases, $R_{\text {min }}$ and $\delta_{\text {cr }}$ both decrease. But when the absorbing layer's thickness is greater than 10 meshes, $R_{\min }$ and $\delta_{\text {cr }}$ have vibration. When the absorbing layer's thickness is 14 , the reflectivity has maximum value. When the absorbing layer's thickness is 20 , $R_{\text {min }}$ get minimum value 0.002 and the corresponding $\delta_{\text {cr }}$ is 1.1. Thus, increase of the absorbing layer's thickness can raise the absorbing effect and reduce reflectivity. But when the thickness increases to some extent, for example 10 meshes, the increase of the thickness has limit effect on the absorbing effect.

\section{ABSORBING EFFECT OF $\delta$ NONLINEARLY DIS- TRIBUTION}

The former study is based on the assumption that $\delta$ varies in the absorbing layer linearly. In fact, the media's variation only needs to be gradually changed and linearity is one choice. Assuming that the media's parameter's variation is second, third or fourth order distribution in the absorbing layer, that is, $\delta=\delta_{\max }(r / d) n$, where $d$ the absorbing layer's thickness,$r$ is the distance to cut off boundary, $\delta_{\max }$ is loss tangent in cut off boundary, $n$ is $2,3,4$ respectively. We study absorbing effect of different $n$ and the corresponding $\delta_{\text {cr }}$.

When $\delta$ is second power distributed, $R_{\min }$ and the corresponding $\delta_{\text {cr }}$ versus thickness are in Fig. (8).

In Fig. (8), when $\delta$ is second power distributed, $R_{\min }$ is smaller than linearly distribution and the corresponding $\delta_{\text {cr }}$ is a little greater. When the absorbing layer's thickness is more than 15 meshes, $R_{\min }$ and the corresponding $\delta_{\text {cr }}$ vary little versus the thickness. Thus, when the absorbing layer's thickness is 15 , the PML's absorbing performance reaches the limit and thickness increase doesn't improve the absorbing effect seriously. 


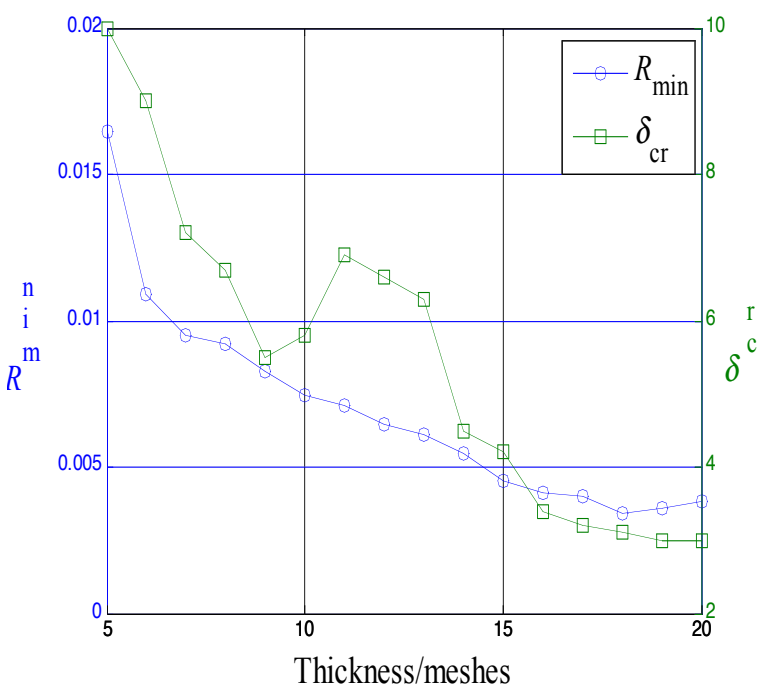

Fig. (9). $R_{\min }$ and $\delta_{\text {cr }}$ when $\delta$ is third power distributed.

When $\delta$ s third power distributed in the absorbing layer, $R_{\text {min }}$ and the corresponding $\delta_{\text {cr }}$ versus thickness is in Fig. (9).

From Fig. (9), when $\delta$ in the absorbing layer is third power distributed, $R_{\min }$ is somehow the same with second power distribution. When the thickness is bigger than 15 meshes, increase of absorbing effect is little.

Study the influence of absorbing layer's thickness on the absorbing effect. For higher power distribution of $\delta, R_{\text {min }}$ decreases via increasing of absorbing layer's thickness. When absorbing layer is thicker than 15 meshes, $R_{\text {min }}$ decreases little. In practical application, 15 layer's thickness can nearly reach the limitation of PML's potential. High power distribution of $\delta$ should be used, but the right $\delta_{\max }$ should be chosen for best absorbing performance.

$R_{\min }$ and corresponding $\delta \mathrm{cr}$ of 15 meshes and 20 meshes thickness is in Table 2.

Table 2 can be a reference when setting PML absorbing layer's parameter. When there is no special demand, PML layer's thickness can be set as 15 meshes. If memory and simulating time permit, absorbing layer's thickness can be set as 20 meshes and in this situation when $\delta$ varies in third or fourth power, the absorbing effect get better.

\section{CONCLUSION}

Based on this paper's calculation result and analysis, we get some conclusion about PML's thickness and parameter's distribution.

1) For different absorbing layer's thickness and $\delta$ 's distribution in it, there is best loss tangent $\delta_{\text {cr }}$ which will result the best absorbing effect. When setting absorbing layer's parameter, $\delta_{\max }$ cannot smaller than $\delta_{\text {cr }}$, but can a little bigger than $\delta_{\text {cr }}$.
Table 2. Absorbing effect of different $\delta$ distribution.

\begin{tabular}{|c|c|c|c|c|c|}
\hline $\begin{array}{c}\text { Thickness / } \\
\text { Meshes }\end{array}$ & & $\begin{array}{c}\text { First } \\
\text { Power }\end{array}$ & $\begin{array}{c}\text { Second } \\
\text { Power }\end{array}$ & $\begin{array}{c}\text { Third } \\
\text { Power }\end{array}$ & $\begin{array}{c}\text { Fourth } \\
\text { Power }\end{array}$ \\
\hline \hline \multirow{2}{*}{15} & $R_{\min }$ & 0.0111 & 0.0047 & 0.0045 & 0.0051 \\
\cline { 2 - 6 } & $\delta_{\mathrm{cr}}$ & 1.7 & 2.5 & 4.2 & 7.1 \\
\hline \multirow{2}{*}{20} & $R_{\min }$ & 0.0061 & 0.0044 & 0.0036 & 0.0036 \\
\cline { 2 - 6 } & $\delta_{\mathrm{cr}}$ & 1.1 & 2.5 & 3.0 & 4.6 \\
\hline
\end{tabular}

2) When $\delta$ 's distribution in the absorbing layer is linearity, the absorbing effect is not good as higher power distribution. When $\delta$ distributes in second, third or fourth power, better absorbing effect can be get.

3) The thicker of the absorbing layer, the better effect of the absorbing effect. In practical application, 15 meshes thickness in absorbing layer can reach PML's absorbing effect potential and then the increase of the thickness can barely improve the absorbing effect.

4) When the second power distribution was used, loss tangent in the cut off boundary can be assigned value 2.5. When better absorbing effect is needed, the absorbing layer's thickness can be set 20 meshes. In this situation, $\delta$ should use third or fourth power distributed and $\delta_{\text {cr }}$ should be set as 3.0 or 4.6 respectively.

\section{CONFLICT OF INTEREST}

The authors confirm that this article content has no conflict of interest.

\section{ACKNOWLEDGEMENTS}

Declared none.

\section{REFERENCES}

[1] S.K. Aggarwal, L.M. Saini, and A. Kumar, "Electricity price forecasting in deregulated markets: A review and evaluation", Int. J. Elec. Power vol. 31, no. 1, pp. 13-22, Jan. 2009.

[2] Y. Lixia, W. Yijun, X. Yingtao, and W. Gang, "Modified uniaxial perfectly matched layer absorbing boundary condition for anisotropic dispersion media", High Power Laser And Particle Beams, vol.23, no. 1, pp.156-160, 2011.

[3] W. Qian, and H. Seng-Tiong. "Implementation of perfectly matched layer boundary condition for finite-difference timedomain simulation truncated with gain medium", J. Lightwave Technol., vol. 29, no.10, pp. 1453-1459, 2011.

[4] O. Ramadan, "Systematic split-step perfectly matched layer formulations for modeling dispersive open region finite difference time domain applications", IET Microw. Antenna. P., vol.5, no. 9, pp. 1062-1066, 2011.

[5] C.M. Choi, and S. Sun. "Simulation of axon activation by electrical stimulation applying alternating-direction-implicit finite-difference time-domain method", IEEE Trans. Magn., vol.48, no. 2, pp. 639642, 2012.

[6] C. Zhan, M. Yanyun, S. Fuqiu, Y. Yan, O. Jianming, and Z. Hongbin, "Implementation of PML absorbing boundary in 2D3V PIC simulation", High Power Laser Particle Beams, vol. 22, no. 5, pp. 1013-1020, 2010. 
[7] Y. Bo, Z. Bi-Hua, G. Cheng, S. Li-hua, C. Bin, and C. Hai-Lin, "Using a two-step finite-difference time-domain method to analyze lightning-induced voltages on transmission lines", IEEE Trans. Electromagn. Compat., vol. 53, no. 1, pp. 256-260, 2011

[8] L. Jiangfan, X. Xiaoli, and W. Guobin, "Simulation of electromagnetic wave propagation through plasma sheath using the movingwindow finite-difference time-domain method", IEEE Trans. Plasma Sci., vol. 39, no. 3, pp. 852-855, 2011

[9] L. Yifeng, and W. Shengxiang, "Convolution perfectly matched layer introduced in FE calculation of 2D acoustic wave in absorptive fluid media", J. Vib. Shock, vol. 31, no. 6, pp. 166-171, 2012.

[10] T. Ohtani, and Y. Kanai, "Optimal Coefficients of the spatial finite difference operator for the complex nonstandard finite-difference time-domain method", IEEE Trans. Magn., vol. 47, no. 5, pp. $1498-1501,2011$.
[11] F. deshan, and X. Yuan, "Three dimensional GPR numerical simulation of full wave field based on UPML boundary condition of ADI-FDTD”, J. Cent. South. Univ. T., vol. 42, no. 8, pp. 23632371, 2011.

[12] V.D Santis, S. Cruciani, M. Feliziani, and M. Okoniewski, "Efficient low order approximation for surface impedance boundary conditions in finite-difference time-domain method", IEEE Trans. Magn., vol. 48, no. 2, pp. 271-274, 2012.

[13] Y. Lixia, L. Qing, Y. Pingping, and Gang, "A novel 3D nonsplitted field perfectly matched layer absorbing boundary condition in FDTD computation", Chin. J. Radio Sci., vol.26, no. 1, pp. 6772, 2011.

[14] Y. Wang, H. Xia, and Q. Cao, "Stability and dispersion analysis of the finite-difference time-domain algorithms in modeling the earthionosphere system", IET Microw. Antenna. P., vol. 5, no. 4, pp. 476-481, 2011.

(C) Ji and Liu et al.; Licensee Bentham Open.

This is an open access article licensed under the terms of the Creative Commons Attribution Non-Commercial License (http://creativecommons.org/licenses/by-nc/3.0/) which permits unrestricted, non-commercial use, distribution and reproduction in any medium, provided the work is properly cited. 